\title{
Analysis of the initial thermal stabilization and air turbulences effects on Laser Tracker measurements
}

\author{
Pablo Pérez Muñoz*, José Antonio Albajez García, Jorge Santolaria Mazo \\ Department of Design and Manufacturing Engineering, University of Zaragoza, Calle María de Luna 3, 50018 Zaragoza, Spain
}

\section{A R T I C L E I N F O}

\section{Article history:}

Received 7 July 2016

Received in revised form 19 September 2016

Accepted 10 October 2016

Available online xxx

\section{Keywords:}

Laser tracker

Metrology

Thermal stabilization

Warm-up period

Turbulence

\begin{abstract}
A B S T R A C T
During the several last years, Laser Trackers have become more common as a measurement tool in the manufacture and assembly of large components such as aircraft wings and ship hulls, as well as for error mapping in coordinate measuring machines and machine tools. Most of these processes cannot be developed in a controlled metrological laboratory but must be implemented directly on a shop floor. Therefore, the process of stabilization of the Laser Tracker has been studied in several experimental tests, and it has been observed that the warm-up time suggested by the manufacturer is not enough. During the first hours of the measurement process two types of thermal errors significantly affect the measurements, causing inaccuracies of between 20 and $80 \mu \mathrm{m}$, depending on the equipment used and the positions of the measured points. These thermal errors are systematic and repeatable; therefore they can be estimated and compensated for each measurement system. Because environmental conditions on a shop floor cannot be controlled, once the Laser Tracker is stabilized, the effects of ambient air in measurements have also been studied, focusing on the effect of turbulent flows on the beam path. It has been observed that this turbulence may cause radial distance drifts on the order of micrometers, deflection of the beam trajectory and signal loss.
\end{abstract}

(C) 2016 Published by Elsevier Ltd

\section{Introduction}

Laser Tracker systems, as portable coordinate measuring machines, have become a notably important metrological tool for industries that must manage large scale components, such as aerospace, wind power, automotive or even machine tool manufacture [1-6]. One common feature of all these sectors is the need to make precise measurements on the shop floor. This is due to the difficulty of moving the large parts involved to an environmentally controlled metrological laboratory.

A tracking interferometer system basically consists of a laser interferometer with two rotary axes that provide the tracking capability, and a target retroreflector (corner-cube or cat's eye type). As a result, this system measures the position of the target on a spherical coordinate system $(\rho, \Theta, \varphi)$. Therefore, the measurement uncertainty of these systems is dependent on the laser system itself, which measures the distance to target $(\rho)$, and the rotary encoders, which provide the angular position $(\Theta, \varphi)$. The angular encoders are widely recognized as one of the main sources of uncertainty [7,8] and the reason of the development of the multilateration techniques [6]. Nevertheless, in the continual effort to attain higher accuracies, some improvements related to distance-measuring uncertainty are still possible.

In a factory workshop without a temperature-controlled environment, the temperature can significantly fluctuate along the day. In [2] is reported an example of an aircraft assembly facility with temperature variations of $8^{\circ}$ over four hours and vertical gradients of $2.2^{\circ}$.

\footnotetext{
* Corresponding author.

Email address: pperezm@unizar.es (P.P. Muñoz)
}

During the aircraft assembly process, if the beginning and the ending temperatures of the measurement survey vary by more than $2.2^{\circ}$, then the survey is considered void and has to be repeated. Environmental conditions have a variety of effects on the measuring process of large components. On one hand, the measured part may present significant dimensional distortions due to temperature variations. These are very difficult to compensate for because they are usually non-uniformly distributed. On the other hand, in non-controlled environments, fluctuations in atmospheric conditions affect the wavelength of the laser beam and, therefore, the stability of the measurement. These effects can be a dominant performance-limiting factor [9]. And lastly, temperature also affects the components of the laser tracker itself: mechanical parts, lenses, the laser source, etc.

There are three major manufacturers of Laser Trackers: Leica Geosystems, Faro Inc. and API. Each manufacturer has its own design and system characteristics [6]. The University of Zaragoza has an API Tracker3 LTS-3000 and a Leica Geosystems LTD600. These two Laser Trackers were used in this work and designated as LT1 and LT2, respectively. The initial thermal stabilization of both Laser Trackers was studied. In order to analyse the effect of air turbulences on the Laser Tracker measurements, only LT1 was used because it allows access to tracking system data. Fig. 1 shows both Laser Trackers and their coordinate systems.

These two Laser Trackers were chosen for their different structural configurations. LT1 has the laser source inside the head, while LT2 has the laser source fixed inside the housing, with a gimballed mirror to direct the laser beam to the retroreflector. The technical specifications of both Laser Trackers are described in Table 1.

There are three main standards concerning performance evaluation of Laser Trackers. ASME B.89.4.19-2005 [10] is the American 


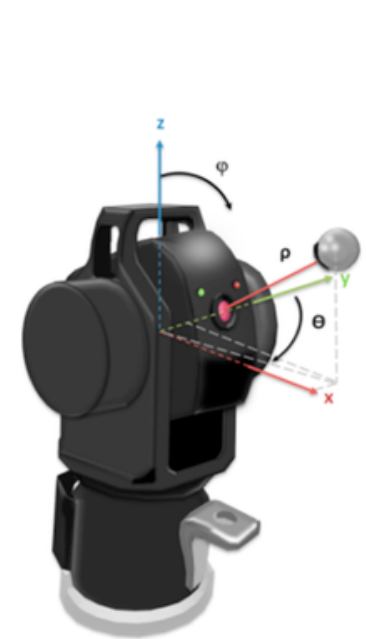

(a)

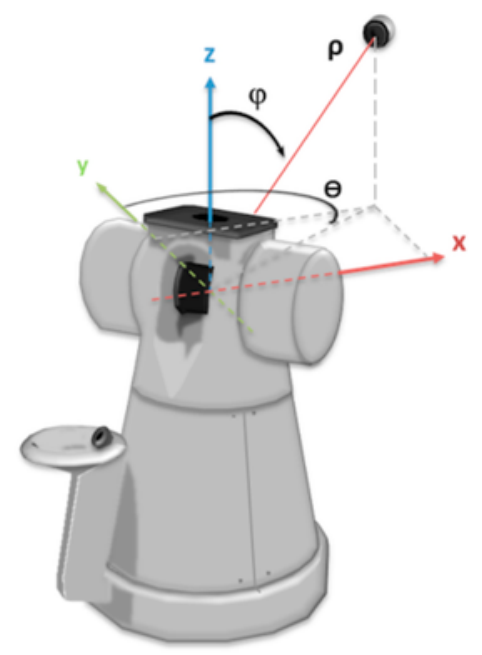

(b)
Fig. 1. Laser Trackers used on the experiments: (a) LT1 with his Cartesian and Spherical coordinate system; (b) LT2 with his Cartesian and Spherical coordinate system.

Table 1

Laser Trackers specifications.

\begin{tabular}{lll}
\hline & LT1 & LT2 \\
\hline Distance Range & $0-30 \mathrm{~m}$ & $0-40 \mathrm{~m}$ \\
Horizontal & $\pm 320^{\circ}$ & $\pm 235^{\circ}$ \\
Vertical & $77^{\circ} /-60^{\circ}$ & $\pm 45^{\circ}$ \\
Distance Resolution (IFM) & $1 \mu \mathrm{m}$ & $1.26 \mu \mathrm{m}$ \\
Distance Accuracy & $\pm 5 \mu \mathrm{m} / \mathrm{m}$ & $10 \mu \mathrm{m} \pm 0.5 \mu \mathrm{m} / \mathrm{m}$ \\
Angular Resolution & $0.07 \mathrm{arc} \mathrm{sec}$ & $0.14 \mathrm{arc} \mathrm{sec}$ \\
\hline
\end{tabular}

National Standard, focused specifically on the use of Laser Trackers as industrial measurement tools. It prescribes methods for the performance evaluation of laser-based spherical coordinate measurement systems and provides a basis for performance comparisons among such systems. These methods and procedures require that the Laser Tracker specifications be accompanied by environmental conditions, including minimum temperature, maximum temperature, and temperature gradients (spatial gradients in ${ }^{\circ} \mathrm{C} / \mathrm{m}$ and temporal gradients in ${ }^{\circ} \mathrm{C} / \mathrm{h}$.). However, the standards methods and procedures do not account for other types of temperature-related errors such as those that might arise from the thermal deformation of the Laser Tracker or the warm-up time. The other two standards, VDI/VDE 2617-10 [11] and ISO 10360-10 [12], do not even consider the effect of air temperature on the laser beam (it is assumed the refractive index is properly estimated). Nevertheless, the concern of manufacturers with these thermal issues can be seen in different patents [13-15] trying to address these problems.

Laser tracker users are also aware of this situation. It can be observed in $[16,17]$ some examples analysing the warm-up time of two different Laser Trackers from an experimental point of view that clearly show the need for longer stabilization times. However, in [16] and [17] no explanation is given for this phenomenon. Therefore, the first part of work here presented focuses on the warm-up behaviour of two different Laser Trackers. It has been evaluated how the increase of temperature when the laser is switched on affects the measurements in two ways: with an 'internal error' and with an 'external error'. The internal error is a consequence of the increase of the temperature inside the Laser Tracker and behaves similarly to the 'death path error' on linear laser interferometers [18], whereas the external error is a consequence of the thermal deformation of the Laser Tracker. If these two errors are not taken into account, the user of the Laser Tracker may be assuming an important error on his measurements.

On the second part of this paper another relevant thermal effect has been addressed. The influence of air and its turbulences on measurements has been studied in order to expose the importance of avoiding heat sources and air flows on the shop floor while the measurement process is being performed.

The environmental conditions influence a laser beam through changes in the refraction index. This results not only in a different value for the measured distance but also in a bending of the ray (a procedure to calculate these effects can be seen in [10]). Currently, the dependency of the refractive index on light wavelength and air temperature, pressure and humidity (and even $\mathrm{CO}_{2}$ content) is well known thanks to such models as the ones proposed by various authors: Edlén [19], Birch and Downs [20,21], or Ciddor [22] which provide theoretical uncertainties of approximately $10^{-7}$ or $10^{-8}$. Therefore, laser systems usually have one weather station to provide the necessary values for these environmental variables (the accuracy of each attribute measured by the weather station can be observed on Table 2). Thus, these equations are especially useful under controlled laboratory conditions where environmental parameters may be considered uniform along the beam path and a single measuring point is representative of the whole environment. Nevertheless, Laser Trackers are used in large workshops where spatial and temporal gradients, which a single weather station cannot detect, are likely to be found. This problem may be compounded by the presence of air turbulence.

Moreover, the signal quality can be greatly impaired in the presence of turbulent air [1]. Thus, from the point of view of Laser Trackers users, the only available solutions consist on the use of fans or air homogenizers (laminar flow) and data averaging over time [1,23]. Nevertheless, this problem has been analysed more deeply in other research areas, such as in geodesy and long range interferometry, where the laser beams have to travel distances in the range of kilometres [24]. Thus, there are several studies about fluctuations in laser beams due to thermal turbulences that are going to be described.

"Turbulence can be described as the random mixing of air particles in the atmosphere due to either rapid or small-scale spatial and temporal temperature-related refractive index fluctuations" [25]. These refractive index fluctuations cause random phase perturbations of the laser beam that can lead to beam distortion. In addition, laser propagation through turbulent media can result in scintillation, beam wander and beam spreading [26]. In 2008, Wang et al. [27] found that in the case of a strong atmospheric turbulence within the light fields, the laser beam loses his Gaussian-shaped distribution as optical vortices are formed. And even after propagation over a sufficiently long-distance for the beam to recover its Gaussian-shaped distribution, the output beam's light is totally incoherent due to the turbulent atmosphere. In $2013 \mathrm{Ji}$ et al. [28] studied changes in the centroid position of laser beams propagating through a turbulent atmosphere and noticed that a cross wind causes a decentred field phase distortion. In $2014 \mathrm{SC}$. Ndlovu et al. [29] found changes in the spatial

Table 2

Weather Station accuracy.

\begin{tabular}{lll}
\hline & LT1 & LT2 \\
\hline Temperature & $\pm 0.3{ }^{\circ} \mathrm{C}$ & $\pm 0.3{ }^{\circ} \mathrm{C}$ \\
Air pressure & $\pm 0.1 \mathrm{KPa}$ & $\pm 0.1 \mathrm{KPa}$ \\
Relative humidity & $\pm 5 \% \mathrm{HR}$ & $\pm 2.5 \% \mathrm{HR}$ \\
\hline
\end{tabular}


intensity profile of a propagating laser beam due to thermal turbulence, even over small distances.

Nevertheless, Laser trackers have the very distinctive capability of tracking the laser beam compared to conventional laser systems. This means that turbulence effects can also be felt not only in the distance range information but also in the angular data provided by the encoders.

Their tracking capability is possible due to a 2D Position Sensitive Detector (PSD) that provides feedback signal to the rotatory motors control [30]. This keeps the returned signal of the retro-reflector in the centre of the PSD and, as a consequence, the light beam also ends centred into the retro-reflector. Optical Position Sensitive Detectors are a kind of photodiodes (1D and 2D) which outputs are proportional to the centroid position of a light spot projected on their surface. The position information is calculated from the relative magnitudes of a few photocurrent signals provided by the PSD. PSDs are widely used in commercial and industrial applications where low-cost or high-speed position sensing is needed [31]. The information provided by the Laser Tracker PSD in our experiments has been used to see how turbulences cause a deflection of the laser beam.

Turbulence causes the beam to blur. The more divergent the beam becomes, the less light falls onto the reflector, and less light is therefore returned to the Position Sensitive Detector, which in the end can no longer detect the reflector signal [30]. This loss of the beam signal may occur because the beam's fluctuations are faster than the Laser Tracker system can follow. The magnitude of these fluctuations can be seen in the magnitude of the root-mean-square error $(\sigma)$ of the measurements.

Since both sources of error (thermal stabilization and air turbulences) have a non-negligible effect on measurements and their uncertainty, especially when the measurement process is conducted on a shop floor where environmental conditions are not controlled, both of these sources of error should be calculated and taken into account.

\section{Warm-up behaviour}

\subsection{Internal error}

Laser Trackers become ready to work after a warm-up process of approximately 15 to $20 \mathrm{~min}$ which allows the laser source to stabilize. Nevertheless, we found that users should wait for at least two hours or even longer to obtain stable measuring data $[16,17]$. In order to determine the real warm-up time of each instrument, the retrore- flector was placed on the birdbath nest (a location where the interferometer distance is set based on previous calibration) as shown in Fig. 2(a). Then, output data were sampled just after the equipment was prepared with the settings in Table 3 and a frequency of one point each $30 \mathrm{~s}$. For $270 \mathrm{~min}$ data were collected in a metrological laboratory with a temperature of $20^{\circ} \mathrm{C} \pm 1{ }^{\circ} \mathrm{C}$. The Variation in radial distance is represented in Fig. 2(b).

After several tests, it was observed that this phenomenon is repeatable and a model is proposed for each system. The radial distance can be fitted according to this general equation:

$$
\rho(t)=A+B \cdot\left(1-e^{-t / \tau}\right),
$$

Where $A$ is the initial value of $\rho$ in the birdbath nest (defined by the manufacturer) in millimeters, $\boldsymbol{B}$ is the maximum value of the radial distance variation in millimeters, $t$ is the instant of time of the measurement in minutes and $\tau$ is the time constant in minutes. For LT1 the equation modeled is

$$
\rho_{L T 1}(t)=159.798+0.019 \cdot\left(1-e^{-t / 30}\right),
$$

For LT2 the equation is modeled as

$$
\rho_{L T 2}(t)=154.999+0.075 \cdot\left(1-e^{-t / 90}\right)
$$

Fig. 2(b) shows mean variation on time of the $\rho$ coordinate (radial distance) of the birdbath nest for both Laser Trackers and the variation of warm-up curves calculated with Eqs. (2) and (3). In exponential equations such as this, $95 \%$ of the final value is reached at time $3 \tau$. This finding means that to obtain stable results, it is necessary to wait 90 min for LT1 and 270 min for LT2. Otherwise, data collected at different times will have some variation. This variation in the radial coordinate should not be neglected, as it has values of 0.019 millimeters for LT1 and 0.075 millimeters for LT2 when stabilized.

This measurement error in the radial component, which occurs during the first hours after the laser source of the Laser Tracker is turned on, is due to the temperature increase inside the housing, which causes expansion of the distance between some components, such the lenses or beam splitter. However, this 'internal error', caused by a thermal drift of the components inside the housing, is an
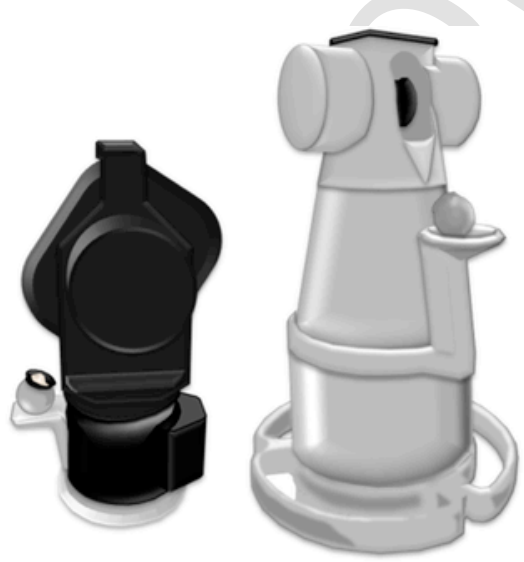

(a)

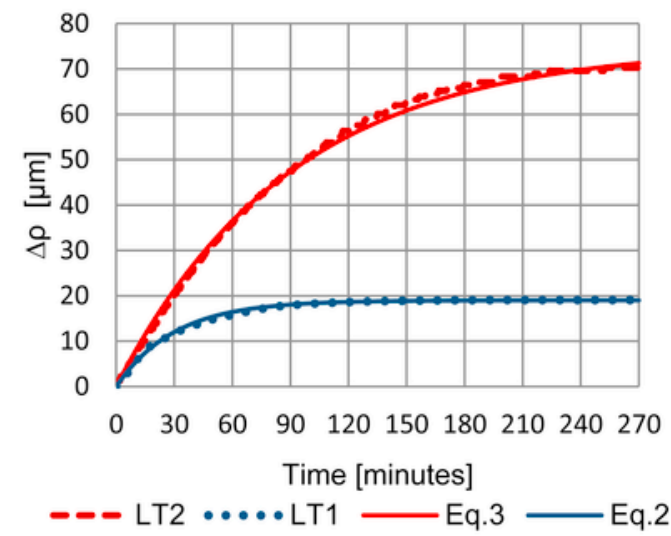

(b)

Fig. 2. Warm-up test: (a) Configuration of the warm-up test; (b) Variation on time of radial distance from the origin of coordinates to the birdbath nest of each Laser Tracker. 
Table 3

Experiment settings.

\begin{tabular}{ll}
\hline Retroreflector & SMR 1.5, \\
\hline Samples per point & $200 \mathrm{samples} /$ point \\
Sampling frequency & $100 \mathrm{~Hz}$ \\
\hline
\end{tabular}

offset that increases along time and only can be corrected by rebooting the Laser Tracker using the function 'Measure Home Position' when the system has thermally stabilized.

\subsection{External error}

The internal heat does not only affect the components inside the tracker, but it also affects the external housing, increasing its temperature and causing thermal dilatation in its structure and, depending on the configuration of the Laser Tracker, a displacement of its origin of coordinates. This effect could be considered as an 'external error' that depends on the position of the heat source. In the case of LT1 the heat source is inside the Laser Tracker head and coincides with the theoretical intersection of the axes of rotation. Therefore the gimbal point remains steady. Nevertheless, since LT2 has its heat source in the housing (under the Laser Tracker head) when the Laser Tracker is switched on, the temperature inside the housing increases and thermal expansion causes the structure to displaces the head and the gimballed mirror up, altering the origin of coordinates of the Laser Tracker.

Temperature sensors were placed on the housing at the positions denoted as $\mathrm{m} 0, \mathrm{~m} 1, \mathrm{~m} 2$ and $\mathrm{m} 3$ (see Fig. 3(a)). During the thermal study of the housing, the air temperature was $21,7^{\circ} \mathrm{C}$, but as seen in Fig. 3(b), during the warm-up period defined by the manufacturer, the housing temperature rises $0.4^{\circ}$, while during the calculated warm-up period the housing temperature rises, depending on the point, between 3 and $4^{\circ}$. To verify that this temperature increase causes expansion of the structure of LT2, an experiment was performed with the settings from Table 3 and the configuration shown in Fig. 4(a).

The first step is to turn on LT1 and wait almost 90 min until the unit is fully warmed up in order to minimize errors. In one set of experiments the retroreflector was fixed on the birdbath nest of LT2 and in other set of experiments the retroreflector was fixed above the LT2 head as shown in Fig. 4(a). LT2 is turned on and during its warm up period LT1 measures the variation of these fixed once per minute. The variation of the height of those points (the $\mathrm{Z}$ coordinate in the Cartesian system of coordinates) is represented in Fig. 4(b).

It can be observed that during the first $120 \mathrm{~min}$ of the test the birdbath nest and the upper surface of the LT2 rose $60 \mu \mathrm{m}$. After another $150 \mathrm{~min}$ both points were stabilized, with the birdbath nest

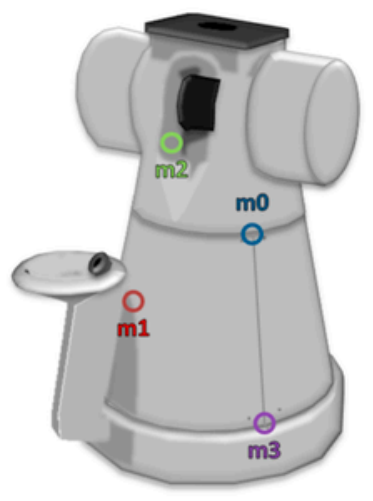

(a)

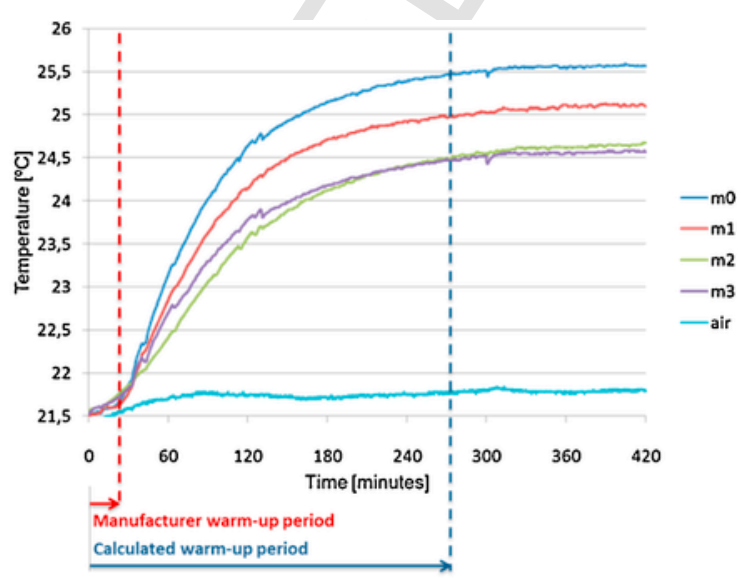

(b)

Fig. 3. LT2 thermal study during warm-up tests: (a) Position of the temperature sensors; (b) Temperature of the housing.

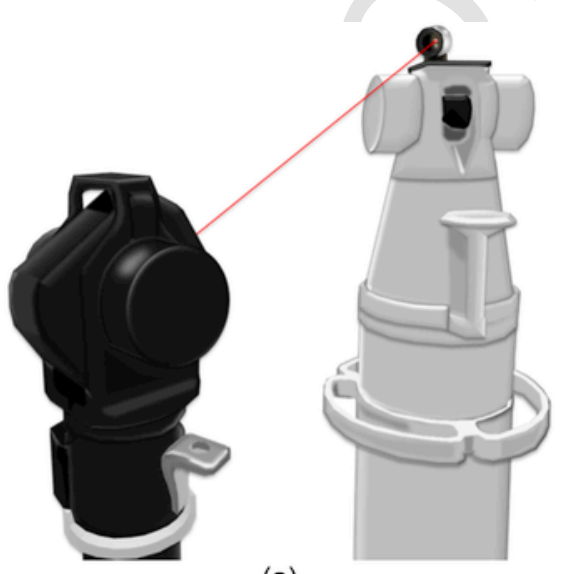

(a)

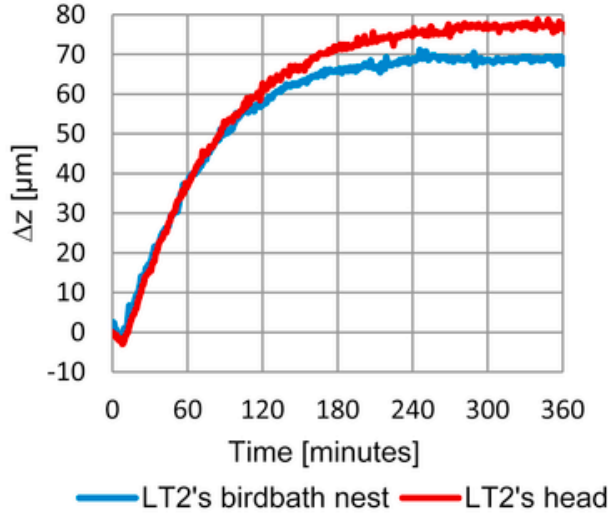

(b)

Fig. 4. Structure dilatation test: (a) Configuration of the test; (b) Variation on time of the Z-coordinate in a Cartesian coordinate system of two points of LT2 measured with LT1. 
reaching a displacement of $70 \mu \mathrm{m}$ and the upper surface of LT2 a displacement of $77 \mu \mathrm{m}$. The $7 \mu \mathrm{m}$ of difference between the upper surface and the birdbath nest is due to the direction of the thermal expansion on the $+Z$-axis. This displacement difference would generate an error in the internal offset that the Laser Tracker is unable to detect or correct.

It should be noticed in Fig. 4(b), that after the warm-up period (20 min after the laser source is turned on), at the moment when the measurement process can be started, both points on LT2 have risen only $5 \mu \mathrm{m}$ due to thermal expansion (which represents around $7 \%$ of the final value) and from then until the moment when LT2 stabilizes, the upper surface of LT2 rises $72 \mu \mathrm{m}$.

\subsection{Correction model of the warm-up behaviour}

\subsubsection{Configuration and data collection}

In order to observe how the errors caused by internal heat affects the Laser Tracker's measurements, a series of fixed bases were placed at different distances and heights over a Coordinate Measuring Machine in a metrological laboratory. The four points measured are designated $\mathrm{A}, \mathrm{B}, \mathrm{C}$ and $\mathrm{D}$, they are on the same straight line to minimize angular errors, but at different radial distances and at different heights. These different distances (A, B and C) and heights (D) are shown in Fig. 5(a). The initial radial distances ( $\rho$ coordinate) of each point are $1352.205 \mathrm{~mm}$ for point A, $1799.363 \mathrm{~mm}$ for point $\mathrm{B}, 2402.274 \mathrm{~mm}$ for point $\mathrm{C}$ and $1658.840 \mathrm{~mm}$ for point $\mathrm{D}$. It should be noted that bases $\mathrm{A}, \mathrm{B}$ and $\mathrm{C}$ have a negative $\mathrm{Z}$-coordinate on the Cartesian coordinate system because they are below the origin of LT2's coordinate system, whereas the base $\mathrm{D}$ has a positive $\mathrm{Z}$-value because it is above the LT2's origin.

The test was performed with the settings shown in Table 3 in a metrological laboratory with an air temperature of $20^{\circ} \mathrm{C} \pm 1{ }^{\circ} \mathrm{C}$. One radial distance value for each point $\mathrm{A}, \mathrm{B}, \mathrm{C}$ and $\mathrm{D}$ are collected every $5 \mathrm{~min}$. After the first $95 \mathrm{~min}$ the retroreflector is brought to the nest and the function 'Measure Home Position' was launched to reset the interferometer, and, after that, distance values for A, B, C and D were taken for another $15 \mathrm{~min}$, resetting the interferometer again before collecting new data. That means that during the first $95 \mathrm{~min}$ the Laser Tracker is producing both types of errors discussed above, internal errors and external errors. However, during the last $15 \mathrm{~min}$ of the experiment, since the Laser Tracker is reset before each measurement, the internal error is corrected and the Laser Tracker is producing only external errors.

The data show that all measurements increase over time, and at the moment when the Laser Tracker is reset, bringing the retroreflector to its birdbath nest (at minute 95 of this experiment), all of the measurements decrease by $45 \mu \mathrm{m}$, which is exactly the value of the internal error of the $\rho$ coordinate in the birdbath nest in minute 95 as seen in Fig. 2(b) for LT2. However, even after applying the correction for internal error, there is a gap of $35 \mu \mathrm{m}$ between A and D. That is because the Laser Tracker is suffering thermal dilatation and external error is not being corrected.

\subsubsection{Correction model}

In this experiment, correction is applied in two steps. First, the external error induced by the thermal expansion of the LT2 housing will be removed for all the data. The second step consists of a correction of the internal error for the first 95 min of the experiment because in the last 15 min this error is corrected when the interferometer is been rebooted.

The $\rho$ coordinate can be calculated as

$$
\rho=\sqrt{\left(x-x_{0}\right)^{2}+\left(y-y_{0}\right)^{2}+\left(z-z_{0}\right)^{2}},
$$

Where $\mathrm{x}_{0}, \mathrm{y}_{0}$ and $\mathrm{z}_{0}$ are the Cartesian coordinates of the origin. The Laser Tracker assumes that the origin is fixed and each coordinate is equal to zero. Thus for the Laser Tracker the radial distance is always

$$
\rho=\sqrt{x^{2}+y^{2}+z^{2}}
$$

However, with the experiment presented in section 2.2 (Fig. 4), it can be observed that the Laser Tracker expands on the $+\mathrm{Z}$-axis and thus the origin of coordinates shifts upwards.

The first step in the correction involves calculating the new value of the $\rho$ coordinate for each point $\left(\rho^{\prime}\right)$ at each moment considering that the origin of coordinates of LT2 is moving on the $+\mathrm{Z}$-axis due to dilatation of the housing, as seen in Fig. 4(b). To correct for this thermal expansion we fix the origin of coordinates subtracting the value of the height of expansion. Next, the new value of the $\rho$ coordinate is calculated as

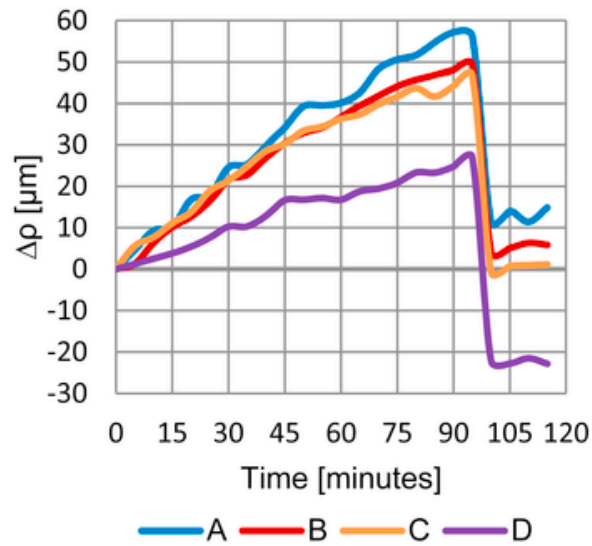

(b) 


$$
\rho^{\prime}=\sqrt{x^{2}+y^{2}+\left(z-z_{L T 2}\right)^{2}},
$$

Where $\mathrm{z}_{\mathrm{LT} 2}$ is the value at each instant of the height to which the origin of coordinates has risen. The negative sign in this equation is present because this height is being subtracted to maintain the origin of coordinates at its initial position.

Fig. 6 shows the new $\rho$ values calculated for each instant of time. Thus, the 'external error' caused by thermal dilatation of the housing has been corrected. Now, in Fig. 6 we can see the $\rho$ coordinates of the four points over time but with the 'internal error' uncorrected for the first $95 \mathrm{~min}$ of the test. The reset of the interferometer which was performed before the data were collected, corrected this error caused by dilatation of the internal components during this last $15 \mathrm{~min}$.

Therefore in the second step the 'internal error' is removed. For this experiment, this correction will be applied only on the values for the first 95 min with this equation:

$$
\rho^{\prime}=\rho^{\prime}-e_{i n t}
$$

Where $\rho$ ' is the $\rho$ coordinate calculated using Eq. (6) and $\mathrm{e}_{\text {int }}$ is the internal error that in this case corresponds with the LT2 values in Fig. 2(b). Fig. 7 shows the $\rho$ " values for each point (after applying the two steps corrections) and $\rho$ values (before applying the corrections):

We observe that before applying the two step correction, the variations in the $\rho$ coordinate were different depending on the position of the point measured but after applying the corrections all the points have a similar behaviour, which is more stable and consistent.

It is important to know the behaviour of the Laser Tracker during its warm-up period if measurements at different moments in time are going to be compared, or measurements taken during this period are to be used. Therefore it is advisable for Laser Tracker users to model their warm-up curve, Eq. (1), and to take into consideration that depending on its design, the Laser Tracker's coordinate origin may suffer a displacement that significantly affects the value of measurements.

\section{Effects of air on measurements}

In a typical laboratory or workshop, the effects of turbulence on commercial straightness or angle interferometers can be severe. In

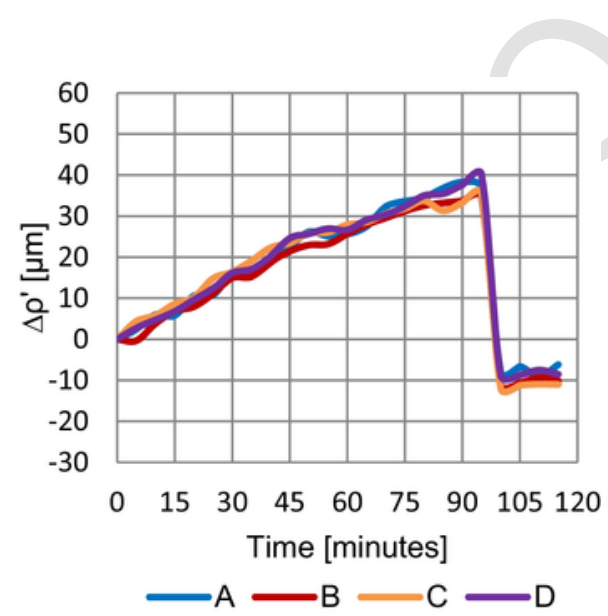

Fig. 6. $\rho$ ' calculated after applying the correction for thermal expansion.

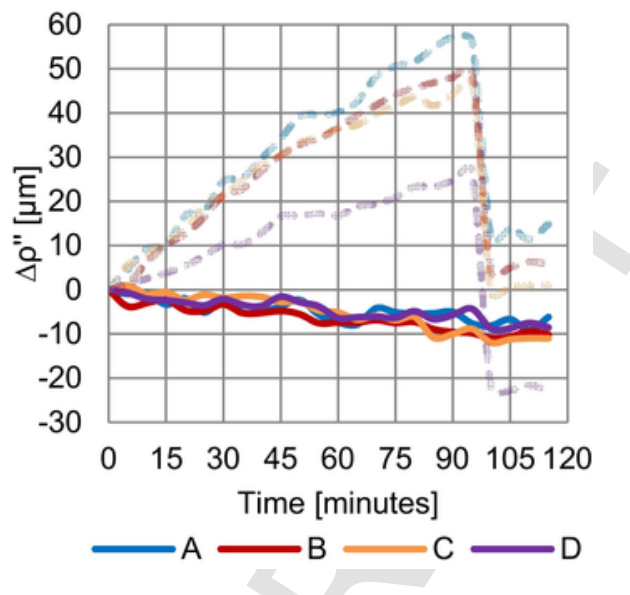

Fig. 7. $\rho$ " calculated after applying the two step corrections.

many cases the only correction possible is data averaging. For path lengths that are not too long, the use of fans or air homogenizers can greatly reduce the effect of turbulence [1], but if a stream of hot air passes through the beam path it will affect the measurements.

To quantify the effects of a turbulent stream of hot air, our equipment was set up as shown in Fig. 8. Although experiments have been developed at different distances, this paper provides the results of tests carried out with a radial distance of two meters.

The retroreflector is fixed at a distance of $2 \mathrm{~m}$ from the Laser Tracker. The data are collected with a frequency of $1 \mathrm{~Hz}$ and $100 \mathrm{sam}-$ ples per point. During the first two minutes the data collected are unaffected by the environment, but after that, a heat source with a turbulent flow is turned on, partially altering the beam path. The heat source increases the temperature and, according to Edlén's equation [19], this increase in temperature causes a decrease in refractive index:

$$
n=1+\frac{7.86 \cdot 10^{-4} \cdot p}{273+t}-1.5 \cdot 10^{-11} \cdot h \cdot\left(t^{2}+160\right)
$$

Where $\mathrm{n}$ is the refractive index, $\mathrm{p}$ is the air pressure in $\mathrm{KPa}, \mathrm{t}$ is the temperature of the air in ${ }^{\circ} \mathrm{C}$ and $\mathrm{h}$ is the relative humidity in percentage.

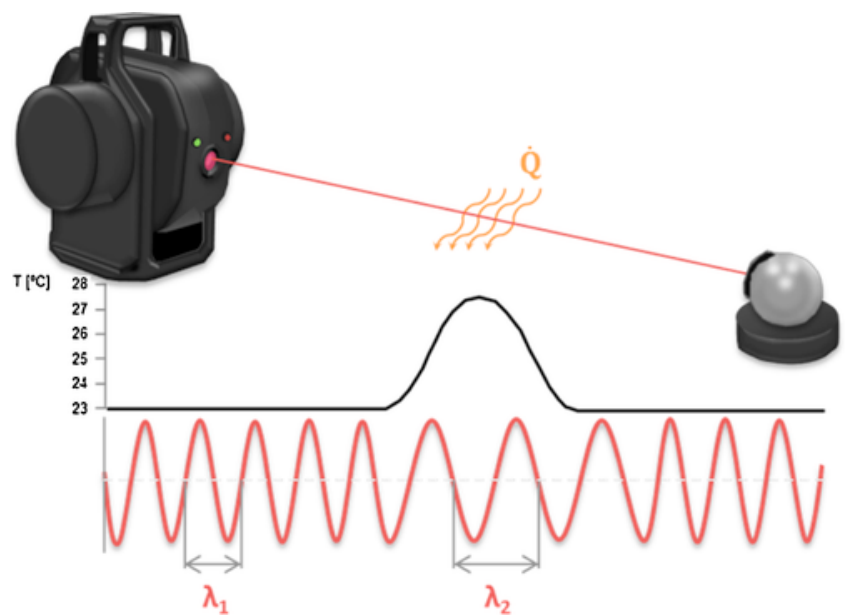

Fig. 8. Configuration of experimental test. 
The refractive index affects the wavelength of electromagnetic radiations according to the relation:

$$
n \cdot \lambda_{\text {air }}=\lambda_{\text {vac }}
$$

Therefore, in the section of the beam path where the temperature has been increased, the refractive index decreases, and consequently the wavelength is greater, as represented in Fig. 8.

Moreover, the Laser Tracker has a weather station to estimate the refractive index of the workshop and assumes that the entire beam path has the same refractive index. As it can be observed in Fig. 9, at second 120 of the experiment, when the heat source is turned on, a part of the beam path suffers a change in its refractive index that the Laser Tracker cannot detect causing a step in the radial distance (see Fig. 9(a)) and an increase in its root-mean-square (standard deviation of the 100 samples) (see Fig. 9(b)).

This radial step is calculated with the following equation:

$$
\rho_{\text {measured }}=\int \frac{n_{i}(x)}{n_{L T}} \cdot \rho_{i} d x
$$

Where $\rho_{\mathrm{i}}$ is the real distance of a section of the beam path $i, \mathrm{n}_{\mathrm{i}}$ is the real refractive index of that section and $\mathrm{n}_{\mathrm{LT}}$ is the refractive index that the Laser Tracker assumes for the entire beam path.

Fig. 9 shows the results of an experiment that ended with a loss of the signal. This loss may have been produced by a dispersion of the laser beam or by a deviation from the geometric straight trajectory of the beam path because while the assumption that light travels in straight line and a constant speed may be valid in a controlled laboratory environment, it is not necessarily valid in a shop floor environment or outdoors where Laser Trackers are often used [32]. In those uncontrolled environments the refractive index suffers variation all along the beam path, which bends the laser beam according to Snell's Law:

$$
n_{1} \cdot \sin \theta_{1}=n_{2} \cdot \sin \theta_{2}
$$

As a result of this curvature, the laser beam is travelling through more space than it would in a perfect straight line. However, this effect has been simulated and calculated and the effect is negligible in terms of distance travelled by the laser (approximately $10^{-5} \mu \mathrm{m}$ per meter of beam path) but not in terms of beam deflection [10].

We observed that when the perturbation on the beam path consists only of an increase in air temperature, without any turbulent air flow, the measurement suffers a variation according to Equation (10). Nevertheless, the laser beam does not suffer any deflection such as that seen in Fig. 9(b) which causes an increase in the root-mean-square error. In such a case, the radial variation can be calculated with Equation (10) and corrected if perturbations on the beam path are measured by temperature and air pressure sensors along the beam path.

With ISO/TS 14253-2 [33] the value of the uncertainty in measurement can be estimated. Therefore, the expected value of the root-mean-square of a measurement can be estimated in order to determine, with this value, if the measurement performed is being taken under the appropriate conditions. Otherwise, if the root-mean-square error is greater than expected, this may be due to vibrations or, as in this case, the presence of a turbulent flow.

Different sources of uncertainty should be taken into account. The main sources of uncertainty are given by the manufacturer and they are:

Absolute accuracy of the Laser Tracker

Repeatability

The values of those contributions are on the Certification Report and for our Laser Tracker can be observed on Table 4.

Other uncertainties that should be taken into account are not given by the manufacturer, one of them is the inaccuracy of the Laser Tracker weather station. The Laser Tracker used for these experiments (LT1) operates with an AT Meteo Station whose accuracy values are shown on Table 2 . To estimate the uncertainty generated by these imprecisions the Monte Carlo method was used with Eqs. (8) and (10). Using the AT Meteo Station's inaccuracies around standard laboratory conditions, which are $20{ }^{\circ} \mathrm{C}, 99 \mathrm{KPa}$ and $50 \% \mathrm{RH}, 10.000$ values are simulated for each attribute measured. The refractive index estimated by the Laser Tracker can be calculated with those data and Eq. (8). Since the true refractive index $\left(n_{i}\right)$ is known, for this simulation the error committed by the Laser Tracker due to the inaccuracy of its weather station can be calculated with Equation (10) as $1.000265156791, \mathrm{n}_{\mathrm{LT}}$ is the refractive index calculated with Eq. (8) and the simulated values that the weather stations can measure, $\rho_{\mathrm{i}}$ is $1 \mathrm{~m}$ to calculate the error per meter. With this data, the error per meter of measurements is shown in Fig. 10.

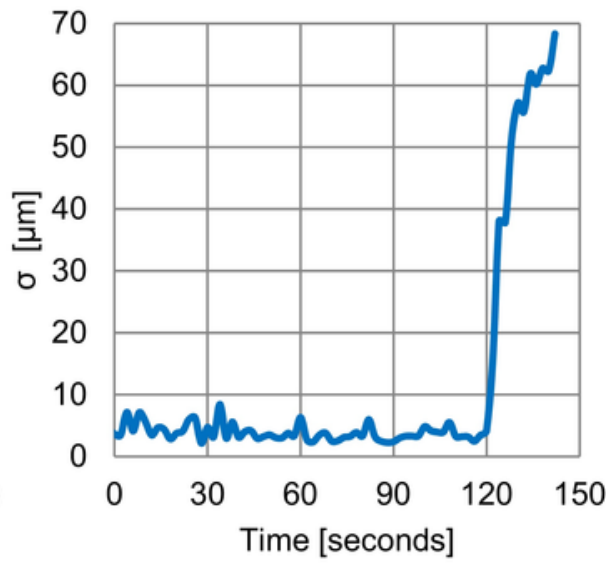

(b)

Fig. 9. Measurements data applying a heat source with turbulent flow at second 120 of the experiment: (a) Variation of radial distance ( $\rho$ ); (b) Variation of root-mean-square ( $\sigma$ ). 
Table 4

Laser Tracker uncertainties sources given by the manufacturer.

\begin{tabular}{ll}
\hline Source & Uncertainty \\
\hline Absolute accuracy & \pm 5 ppm $(2 \sigma)$ \\
Repeatability & $\pm 2.5 \mathrm{ppm}(2 \sigma)$ \\
\hline
\end{tabular}

According to the Guide to the expression of Uncertainty in Measurement (GUM) [34] when the error presents a normal distribution with, in our case, $2 \sigma \approx 0.385 \mu \mathrm{m} / \mathrm{m}$ (see Fig. 10), this value of $0.385 \mathrm{ppm}$ is the uncertainty of the measurement caused by the weather station.

Another source of uncertainty that is not given by the manufacturer that should be taken into account is the error caused by incorrect information regarding the weather conditions along the beam path. That is because the Laser Tracker has information on just one point near to the beam path, but along the path there exist variations in temperature, air pressure and relative humidity. Even for a very stable environment with a temperature gradient of just $0.1{ }^{\circ} \mathrm{C} / \mathrm{m}$, the error over a beam length of $10 \mathrm{~m}$ due to refractive changes would be $5 \mu \mathrm{m}$, while for a more typical $1{ }^{\circ} \mathrm{C} / \mathrm{m}$ the error would be $50 \mu \mathrm{m}$ [35]. Techniques are being developed to compensate for these effects by using multiple frequencies of light [36].

To determine the value of this uncertainty it would be necessary to evaluate the shop floor conditions to estimate the likely variations, and estimate the uncertainty per meter with the Monte Carlo method and Eqs. (10) and (8). There are open source software programs are available for monitoring the environmental conditions at a low cost [37].

The GUM [34] indicates that the combined uncertainty can be calculated as

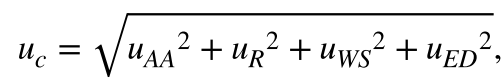

Where $u_{A A}$ is the absolute accuracy uncertainty, $u_{R}$ is the repeatability uncertainty, $\mathrm{u}_{\mathrm{WS}}$ is the weather station uncertainty and $\mathrm{u}_{\mathrm{ED}}$ is the uncertainty due to environmental deviation on the beam path. Taking the values from Table $4, \mathrm{u}_{\mathrm{AA}}$ is $5 \mathrm{ppm}$ and $\mathrm{u}_{\mathrm{R}}$ is $2.5 \mathrm{ppm}$. The value of $u_{W S}$ has been calculated as 0.385 ppm and assuming that $u_{E D}$ is $0 \mathrm{ppm}$ in our experiment because it was conducted at $2 \mathrm{~m}$ in a laboratory with controlled environmental conditions. Using a distribution factor of 0.5 , the combined uncertainty is

$$
u_{c}=\sqrt{2.5^{2}+1.25^{2}+0.193^{2}+0}=2.80 \mathrm{ppm},
$$

The expanded uncertainty $(\mathrm{k}=2)$ would be

$$
U=k \cdot u_{c}=2 \cdot 2.80=5.6 p p m,
$$

In our experiment whose radial distances variations are shown in Fig. 9, the distance between the Laser Tracker and the retroreflector is $2 \mathrm{~m}$, so the expected uncertainty, $U(2 \sigma)$, is $11.2 \mu \mathrm{m}$. Therefore, the value of the root-mean-square $(\sigma)$ for this experiment is expected to be lower.

As can be observed in Fig. 9(b) when the Laser Tracker works in an unaltered environment the root-mean-square error is between $2 \mu \mathrm{m}$ and $8 \mu \mathrm{m}$. However, when heat turbulence is introduced this value increases to almost $70 \mu \mathrm{m}$. This means that of the 100 samples taken by the Laser Tracker for $1 \mathrm{~s}$ for each point, when the heat source is turned on, $95 \%$ of the samples are between $\pm 140 \mu \mathrm{m}$. Moreover, these fluctuations may be sufficiently fast so that the tracking system can no longer follow the signal. Furthermore, angle of arrival fluctuations in the received wave front affect the PSD which suffers from additional precision deterioration due to receiver misfocusing [27].

The deflection can be observed in the data exported from the PSD. Because the beam propagates along the $\mathrm{Z}$-axis, the turbulence causes a deflection in the $\mathrm{X}$ and $\mathrm{Y}$-axes and when the beam is deflected the Laser Tracker tries to re-centre using its motor-encoders.

When there is no perturbation on the beam path and the retroreflector is in a fixed position, the values of PSD X and PSD Y are around $\pm 1 \mu \mathrm{m}$. When the heat source is turned on, however, beam loss does not occur immediately. When the Laser Tracker loses the signal, it loses the ability to track and measure, but the PSD continues to receive a signal. Fig. 11 shows in blue the position of the laser beam as seen by the PSD during the last $0.25 \mathrm{~s}$ before beam loss, and in red the next $0.25 \mathrm{~s}$ after beam loss. Before the signal loss the laser beam deflects $\pm 20 \mu \mathrm{m}$ on both $\mathrm{X}$ and $\mathrm{Y}$ axes, but when the Laser

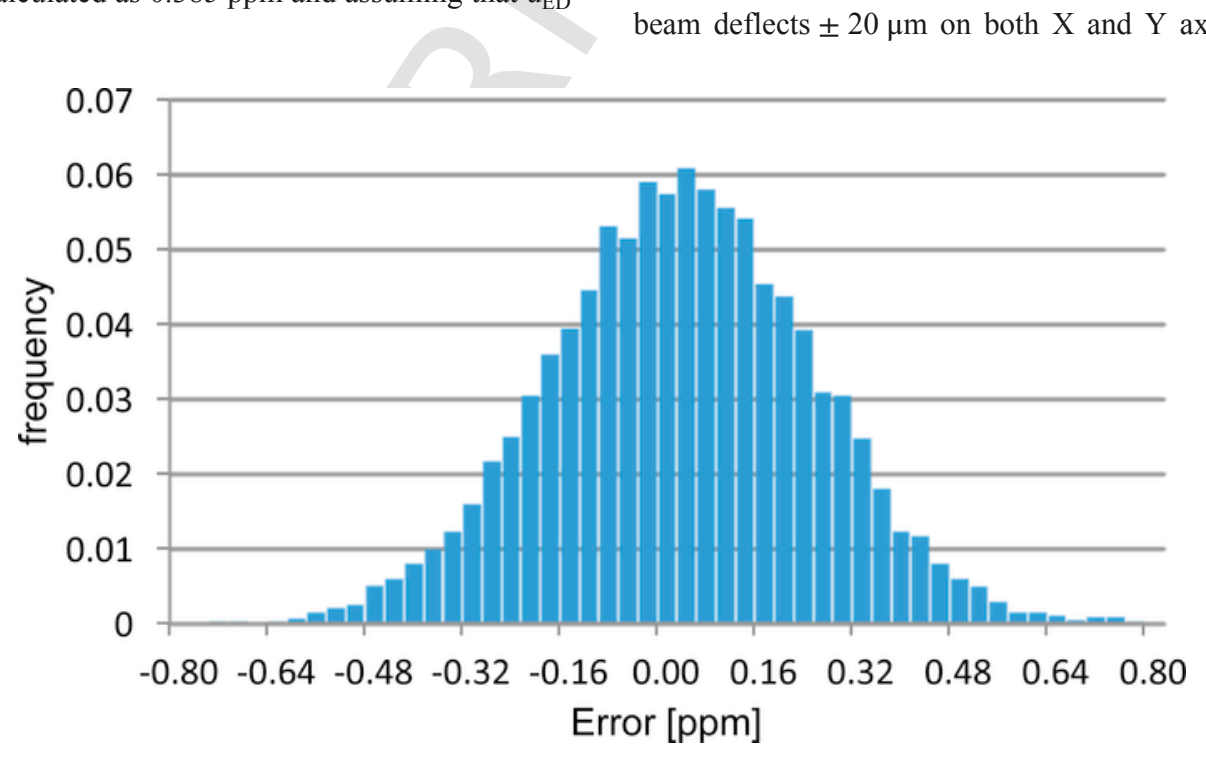

Fig. 10. Radial distance error caused by the inaccuracies of the weather station. 


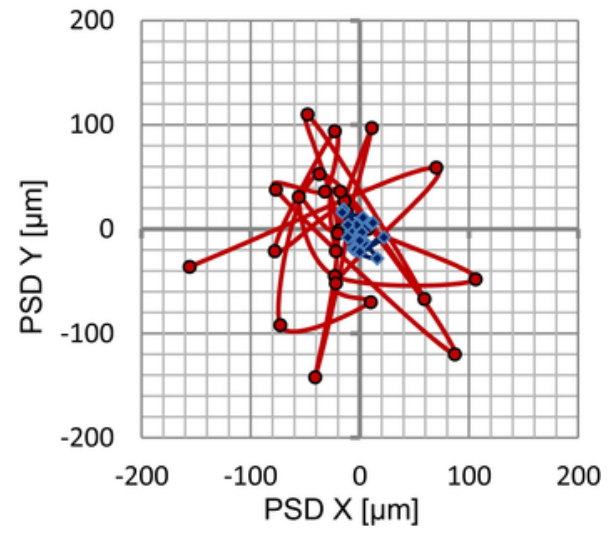

Fig. 11. Laser beam deflection determined by the PSD corresponding to Fig. 9.

Tracker loses the beam signal, the PSD shows deflections around $\pm 100 \mu \mathrm{m}$ on both axes.

Fig. 12 shows the distance between two consecutive samples of the data shown in Fig. 11. The sampling frequency is $100 \mathrm{~Hz}$, so there are $0.01 \mathrm{~s}$ between two consecutive samples. Both Figures show PSD data over $0.5 \mathrm{~s}, 0.25 \mathrm{~s}$ before and after the loss of beam signal. The turbulence, because of its randomness, causes the laser beam to oscillate around the theoretical straight trajectory. When this oscillation is moderate, approximately $20 \mu \mathrm{m}$, the PSD of the Laser Tracker

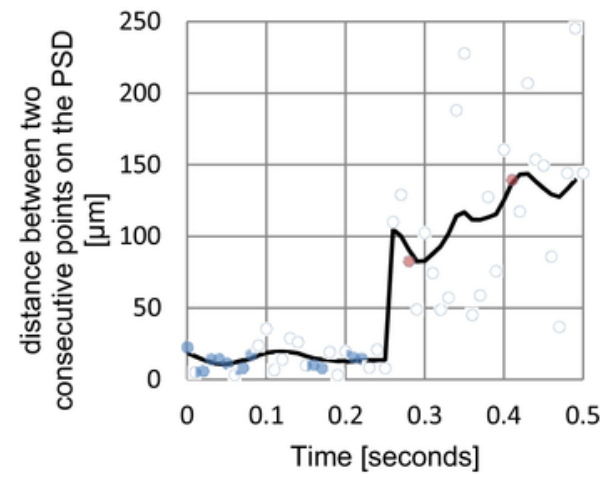

Fig. 12. Laser beam deflection saw by the PSD before and after the beam loss.

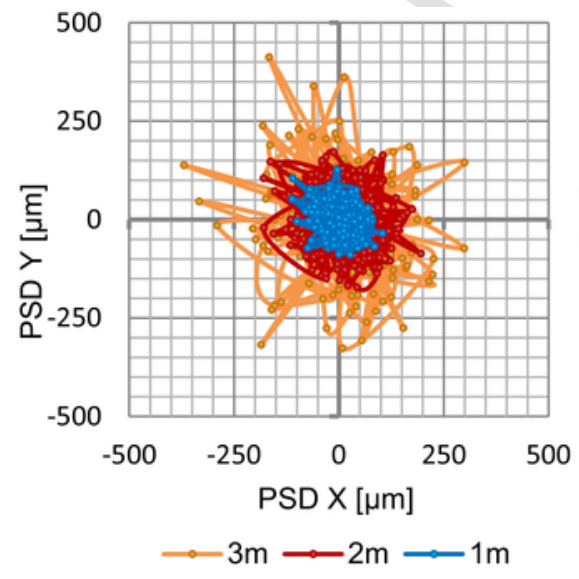

(a) can follow the laser beam without any problem, but when this oscillation increases dramatically to values of $100 \mu \mathrm{m}$ or even more, the PSD loses the signal and data collection is stopped.

Fig. 13(a) shows laser beam deflection for different radial distances using data collected a few seconds after beam loss (approximately 3 s). This deflection can be seen also in Fig. 13(b) which shows the root-mean-square at the moment of the loss of the signal for different distances. We can see that the longer the distance travelled by the laser beam the more it deflects, and thus the signal is lost more easily if any turbulence is present. As Laser Trackers are widely used for high precision measurements of large scale components, it is important to take this phenomenon into account.

\section{Summary and conclusions}

In a high-precision measurement process it is important to avoid as many sources of error as possible to achieve the highest level of accuracy. We have shown that when a Laser Tracker is switched on, the warm-up period indicated by the manufacturer is not sufficient to achieve total stabilization of the Laser Tracker and, depending on the configuration of the equipment, this has an important effect on measurements accuracy. An analysis of the thermal stabilization of two Laser Trackers was conducted. Both Laser Trackers have a manufacturer warm-up period of approximately $20 \mathrm{~min}$, during which time no data can be collected, but after which measurements can be performed. Several experiments demonstrated that this time is insufficient to reach an optimal stabilization of the Laser Tracker. Depending on the specific Laser Tracker, the optimal warm-up period is between an hour and a half and four and a half hours.

During this thermal stabilization time, the temperature inside the Laser Tracker increases and some components, such as lenses, mechanical parts and even the housing expand causing errors that are relevant if measurements from two different instants of time are compared. These errors are systematic and repeatable therefore can be estimated and corrected. Two solutions have been proposed to prevent errors caused by incomplete thermal stabilization. One solution is to wait for the entire warm-up period and reset the interferometer before starting the measurement process. If time constrains prevent a proper warm-up period, the second solution is to model the warm-up curve and the structural dilatation curve (if necessary) and correct the measurements with the model presented in Section 2.3.2. It is advisable to model the warm-up curve of this initial period to understand the

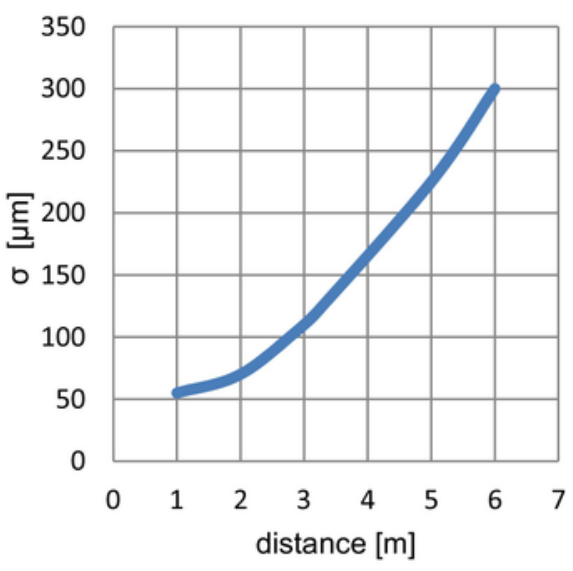

(b)

Fig. 13. PSD data: (a) Laser beam deflection for different distances (1, 2 and $3 \mathrm{~m}$ ); (b) Variation of root-mean-square ( $\sigma)$ at the moment of the beam loss for different distances. 
behaviour of the equipment and in order to reduce the level of uncertainty. Nevertheless, the dilatation of Laser Tracker's structure needs to be further studied to encourage manufacturers to reduce this phenomenon that alters the position of the origin of coordinates and therefore it affects the measurement process. Another consequence of this dilatation is an increase in the distance between the upper surface and the bird bath nest over time $(7 \mu \mathrm{m})$. Thus, the real distance between the gimballed mirror and the bird bath nest is not constant. The effect of this variation on the measurement uncertainty would require further study.

The second part of this paper describes another source of error that may be present on a shop floor, the possible presence of turbulences or flows of hot air across the beam path that can appear because environmental conditions are not controlled. These turbulences modify the wavelength of the laser beam and this leads to a variation in the radial measurement. If the turbulence persists, it can cause a loss of the signal, ending the measurement process. Moreover, we observed, using data from the Position Sensors of the Laser Tracker, that these turbulences cause a random deflection of the trajectory of the laser beam, which significantly increases the uncertainty of the measurement. Therefore, it is advisable to avoid airflows and heat sources while the measurement process is being performed. This can be detected by checking the root-mean-square valued for every measurement, though a real correction procedure will require further research.

\section{Conflicts of interest}

The authors declare no conflict of interest.

\section{Acknowledgments}

This work was supported by the Project of The Secretary of State of Research, Development and Innovation, within the Spanish Ministry of Economy and Competitiveness DPI2013-46979-C2-1-P and the Funds of the scholarship BES-2014-070480.

\section{References}

[1] W.T. Estler, K.L. Edmundson, G.N. Peggs, D.H. Parker, Large-scale metrology - an update, Ann. CIRP 51 (Pt 2) (2002) 587-609.

[2] Muske S, Salisbury D, Salerno R, Calkins J, 747 Data Management System Development and Implementation, In: Presented at the 1999 CMSC Conference and the 2000 Boeing Large Scale Metrology Conference.

[3] F. Härtig, H. Lin, K. Kniel, Z. Shi, Laser Tracker performance quantification for the measurement of involute profile and helix measurements, Measurements 46 (Pt 8) (2013) 2837-2844.

[4] J. Yin, Y. Gao, Pose accuracy calibration of serial five DOF Robot, Energy Procedia 14 (2012) 977-982.

[5] A. Paoli, A.V. Razionale, Large yacht hull measurement by integrating optical scanning with mechanical trackin-based methodologies, Robot. Comput. Integr. Manuf. 28 (2012) 592-601.

[6] S. Aguado, J. Santolaria, D. Samper, J. Velazquez, C. Javierre, A. Fernández, Adequacy of technical and commercial alternatives applied to machine tool verification using Laser Tracker, Appl. Sci. 6 (Pt 4) (2016) 100, http://dx.doi.org/ 10.3390/app6040100.

[7] K.M. Nasra, B. Hughes, A. Forbes, A. Lewis, Determination of laser tracker angle encoder errors. EPJ web of conferences, 16th International Congress of Metrology 77 (2014) http://dx.doi.org/10.1051/epjconf/20147700002.

[8] G. Gassner, R. Ruland, Laser tracker calibration - Testing the angle measurement system, SLAC National Accelerator Laboratory Publication, SLAC-PUB-13476, 2008. December.

[9] Renishaw apply innovation, Application note: Air turbulence effect on measurement stability of the differential interferometer. Available online: http:// resources.renishaw.com/en/details/ application-note-air-turbulence-effects-on-measurement-stability-of-the-differential-interfere cessed on 05.07.16]
[10] ASME B89.4. 19-2005 Performance Evaluation of Laser Based Spherical Coordinate Measurement Systems, Vol. 02, American Society of Mechanical Engineers, New York, NY, USA, 2005. August p. 66.

[11] VDI/VDE 2617 Blatt 10. Accuracy of coordinate measuring machines - Characteristics and their checking - Acceptance and reverification tests of laser trackers.

[12] ISO 10360-10:2016. Geometrical product specifications (GPS) - Acceptance and reverification tests for coordinate measuring systems (CMS) - Part 10: Laser trackers for measuring point-to-point distances.

[13] Patent US 9329028 B2. Spherically mounted retroreflector having an embedded temperature sensor and socket. Faro Tech Inc. 2016.

[14] Patent US 7466401 B2. Self-compensating laser tracker. Faro Tech Inc. 2008.

[15] Patent US 20120320943 (A1). Automated and accelerated warm-up and stability check for laser trackers. Faro Tech Inc. 2012.

[16] R. Sugahara, M. Masuzawa, Y. Ohsawa, Performance test of laser trackers of FARO, Proceedings of the 7th annual meeting of Particle Accelerator Society of Japan (2010) p1232.

[17] G. Gassner, R. Ruland, Instrument test with the new Leica AT401, In: The 11th International Workshop on Accelerator Alignment (IWAA2010), DESY Hamburg, Germany, September 13-17, 2010.

[18] Renishaw apply innovation, Technical white paper: TE329. Environmental compensation of linear laser interferometer readings, 2013. Available online: http://resources.renishaw.com/en/details/

white-paper-environmental-compensation-of-linear-laser-interferometer-readings-53595 [accessed on 05.07.16].

[19] B. Edlén, The refractivity of air, Metrologia 2 (Pt 2) (1966) 71-80.

[20] K.P. Birch, M.J. Downs, An updated Edlén equation for the refractive index of air, Metrologia 30 (1993) 155-162.

[21] K.P. Birch, M.J. Downs, Correction to the updated Edlén equation for the refractive index of air, Metrologia 31 (1994) 315-316.

[22] P.E. Ciddor, Refractive index of air: new equations for the visible and near infrared, Appl. Opt. 35 (9) (1996) 1566-1573.

[23] J. Muelaner, O.C. Martin, P.G. Maropoulos, Metrology enhanced tooling for aerospace (META): strategies for improved accuracy of jig built structures, SAE Aerotech. (2011). 2011-10-18-2011-10-21.

[24] L. Redovniković, I. Ališić, A. Luketić, Influence of lateral refraction on measured horizontal directions, Surv. Rev. 45 (Pt 331) (2013) 285-295, http://dx. doi.org/10.1179/1752270612y.0000000037.

[25] A. Ishimaru, Theory of optical propagation in the atmosphere, Opt. Eng. 20 (1981) http://dx.doi.org/10.1117/12.7972665.

[26] S.M. Augustine, N. Chetty, Experimental verification of the turbulent effects on laser beam propagation in space, Atmósfera 27 (Pt 4) (2014) 385-401.

[27] L.G. Wang, W.W. Zheng, L.Q. Wang, Effect of atmospheric turbulence on propagation properties of optical vortices formed by using coherent laser beam arrays, J. Opt. A Pure Appl. Opt. 11 (6) (2009) http://dx.doi.org/10.1088/ $1464-4258 / 11 / 6 / 065703$.

[28] X. Ji, Y. Baykal, X. Jia, Changes on the centroid position of laser beams propagation through an optical system in turbulent atmosphere, Opt. Laser Technol. 54 (2013) 199-207.

[29] S.C. Ndlovu, N. Chetty, Analysis of the fluctuations of a laser beam due to thermal turbulence, Cent. Eur. J. Phys. 12 (Pt 7) (2014) 466-472.

[30] Leica Geosystems Leica Absolute Tracker AT401 White Paper, 2010. Available online: http://metrology.leica-geosystems.com/en/page_search.htm?area $=45 \&$ search $=$ true $\& \mathrm{q}=\&$ product $=$ Absolute + Tracker + AT $401 \&$ type=Whitepaper\%2FTechnical+Paper [Accessed on 05.07.16].

[31] A. Mäkynen, Position-Sensitive Devices and sensor systems for optical tracking and displacement sensing applications Doctoral thesis, Available online: http:// urn.fi/urn:isbn:9514257804 [accessed on 05.07.16] Faculty of Technology, University of Oulu, Oulu, Finland, 2010.

[32] B. Muralikrishnan, S. Phillips, D. Sawyer, Laser trackers for large scale dimensional metrology: a review, Precision Eng. (2015) http://dx.doi.org/10.1016/j. precisioneng.2015.12.001.

[33] ISO/TS 14253-2 Guide to the estimation of uncertainty in GPS measurement, in calibration of measuring equipment and in product verification 1999.

[34] Joint Committee for Guides in Metrology (JCGM 100:2008). Evaluation of measurement data -Guide to the expression of uncertainty in measurement 2008.

[35] J.E. Muelaner, P.G. Maropoulos, Large volume metrology technologies for the light controlled factory, Procedia CIRP 25 (Pt C) (2014) 169-176.

[36] M. Vorontsov, G. Carhart, R. Gudimetla, T. Weyrauch, E. Stevenson, S. Lachinova, et al., Characterization of atmospheric turbulence effects over $149 \mathrm{~km}$ propagation path using multi-Wavelength laser beacons, In: Advanced Maui orfettic $\$ 00028$ Space Surveillance Technologies Conference, Maui, HI, USA, September 14-17, 2010

[37] A.J. Lewis, M. Campbell, P. Stavroulakis, Performance evaluation of a cheap, open source, digital environmental monitor based on Raspberry Pi, Measurement 87 (2016) 228-235. 